\title{
Influence of Eccentricity and Angular Velocity on Force Effects on Rotor of Magnetorheological Damper
}

\author{
Dominik Šedivý ${ }^{1,}$, Petr Ferfecki ${ }^{2}$, Simona Fialová ${ }^{1}$ \\ ${ }^{1}$ Brno University of Technology, Victor Kaplan Department of Fluid Engineering, 61669 Brno, Czech Republic \\ ${ }^{2}$ VŠB - Technical University of Ostrava, IT4Innovations, 70833 Ostrava, Czech Republic
}

\begin{abstract}
This article presents the evaluation of force effects on squeeze film damper rotor. The rotor is placed eccentrically and its motion is translate-circular. The amplitude of rotor motion is smaller than its initial eccentricity. The force effects are calculated from pressure and viscous forces which were measured by using computational modeling. Damper was filled with magnetorheological fluid. Viscosity of this nonNewtonian fluid is given using Bingham rheology model. Yield stress is not constant and it is a function of magnetic induction which is described by many variables. The most important variables of magnetic induction are electric current and gap width between rotor and stator. The simulations were made in finite volume method based solver. The motion of the inner ring of squeeze film damper was carried out by dynamic mesh. Numerical solution was solved for five different initial eccentricities and angular velocities of rotor motion.
\end{abstract}

\section{Introduction}

Change of fluid properties in working damper is one of possible ways to improve damping ability of damper. However this change has to be very fast. Magnetorheological (MR) fluids are liquids which show fast transition from a liquid to a nearly solid state under the presence of external magnetic fields. Generally, their viscosity depends on magnetic induction and therefor MR fluids belong to non-Newtonian fluids. This special property makes them very good candidates for applications in mechanical systems which require active control of vibrations. Classical examples are shock absorbers or vibration dampers. Shaft vibrations are generated from the imbalance rotor motion which can be caused by assembling or manufacturing inaccuracies. These negative effects can be noticeably minimized if active or semi-active damping elements are installed between the shaft and the stationary part of the rotating machine. Positive parameters of MR fluids in connection with conventional squeeze film dampers provide a possibilities to design a semi-active damping elements. Nowadays the MR fluids are a subject of an intensive experimental and theoretical research. Mathematical models of squeeze film damper were published in works $[2,4]$. A computational simulation was done in article [1]. An interaction between surface wettability and MR fluids was solved in works $[5,6]$. Force effects on squeeze film damper were studied in paper [7].This article continues in research, which was started in work [7].

\section{Methods}

Application of squeeze film damper is shown in Fig. 1. The rotor shaft is attached in bearings which allow its rotation. There is a fixed connection between bearings and housing of bearings by a squirrel spring. Squeeze film damper is consisting of an inner and an outer ring and magnetorheological oil. The primary aim of damper is to eliminate unwanted vibrations. Magnitude of viscosity is controlled by the electric current in electric coil which generates magnetic induction. The domain for numerical simulation was just the place between inner and outer ring.
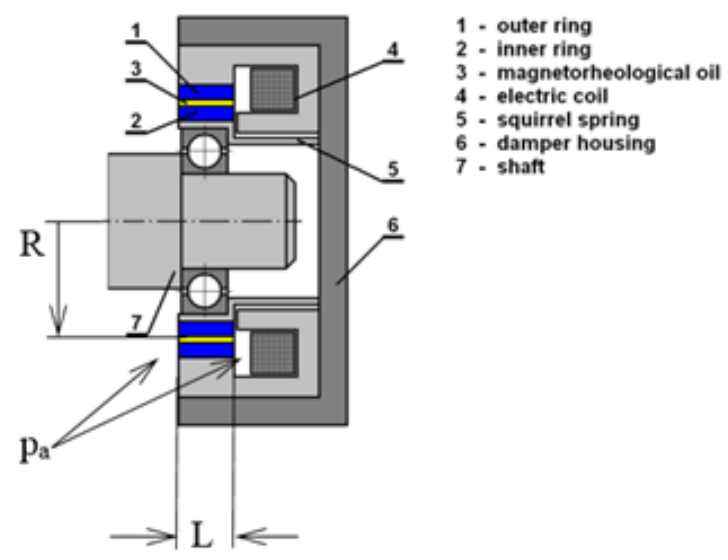

Fig. 1. Apllication of squeeze film damper [3]

The outer ring is a stationary wall and its diameter is 152 $\mathrm{mm}$. The inner ring is a moving wall with $150 \mathrm{~mm}$ diameter and is eccentric placed in relations to outer ring

Corresponding author: Dominik.Sedivy@vutbr.cz 
Motion of inner ring is translate-circular and its amplitude of deviation is variable. Mean value of eccentricity (center of movement) in direction of $\mathrm{x}$ axis was solved for five different values. For every case was maximal eccentricity in direction of $\mathrm{x}$ axis $0.55 \mathrm{~mm}$. The motion for case that mean value of eccentricity is 0.5 $\mathrm{mm}$ and amplitude of deviation is $0.05 \mathrm{~mm}$ is shown in Fig. 2.

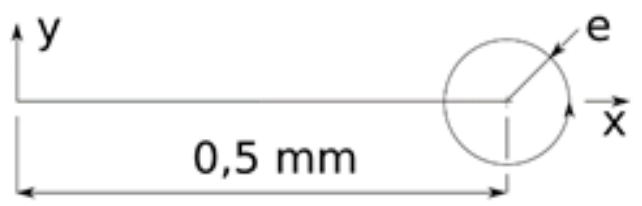

Fig. 2. Eccentric motion

Combinations of deviation amplitudes and mean values of eccentricities are shown in Table 1.

Table 1. Combination of eccentricity and deviation

\begin{tabular}{|c|c|}
\hline $\begin{array}{c}\text { Mean value of eccentricity } \\
{[\mathrm{mm}]}\end{array}$ & $\begin{array}{c}\text { Amplitude of deviation } \\
{[\mathrm{mm}]}\end{array}$ \\
\hline 0.52 & 0.03 \\
\hline 0.51 & 0.04 \\
\hline 0.5 & 0.05 \\
\hline 0.49 & 0.06 \\
\hline 0.48 & 0.07 \\
\hline
\end{tabular}

The real length of the damper is $44 \mathrm{~mm}$. The numerical solution was solved by commercial CFD (computational fluid dynamics) software (ANSYS Fluent). Following boundary conditions were set up for numerical simulation. To obtain lower simulation time, the geometry was cut in the middle of damper length and symmetry condition was used. The outer ring was determined as a stationary wall. At the outlet from damper, a pressure outlet with magnitude of relative pressure $0 \mathrm{~Pa}$ was set. The inner ring of squeeze film damper was defined as a moving wall. Its motion was prescribed by velocities functions for every axis:

$$
\begin{aligned}
& v_{x}=-e \cdot \omega \cdot \sin (\omega \cdot t) \\
& v_{y}=e \cdot \omega \cdot \cos (\omega \cdot t)
\end{aligned}
$$

where $v_{x}$ and $v_{y}$ are components of velocity in direction of $x$ and $y$ axis,

$e$ is amplitude of deviation.

Frequency of inner ring motion is prescribed by its angular velocity $\omega=(60 ; 70 ; 80 ; 90 ; 100) \cdot \pi s^{-1}$. The velocity in direction of $z$ axis was equal to $0 \mathrm{~m} \cdot \mathrm{s}^{-1}$.

Numerical simulations were calculated by using the dynamic mesh. Displacements were small in these solutions, therefore the meshes of computational domains did not have to be remeshed (mesh is redesigned - number of elements are changing during the simulation). Mesh of computational domain has 320000 hexahedral elements (minimum orthogonal quality = 0,888 ; maximum ortho skew $=0,112$; maximum aspect ratio $=18,928)$. Because the mesh was just deformed (stretched and compressed), Smoothnig method was applied, which is included in ANSYS Fluent. Diffusion parameter was defined as Cell-volume and its value was set equal to 0 . The motion of inner ring was prescribed by user defined function (UDF) and inner ring was set as rigid body.

\section{Mathematical model of fluid}

Density of magnetorheological fluid has value $970 \mathrm{~kg}$. $m^{-3}$. For case there is no electric current in the coil, magnetorheological fluids have constant viscosities and the fluid behavior is Newtonian. Electric current in coil generates a magnetic field and the magnetorheological fluid will change its properties. For the mixed viscosity the Bingham rheology model applies which was defined in work [1]. This model was also used in paper [7]. The model is described by equation:

$$
\tau=\tau_{y}+\mu \cdot \dot{\gamma}
$$

where $\tau[\mathrm{Pa}]$ is shear stress which acts upon the element of fluid,

$\tau_{y}[\mathrm{~Pa}]$ is shear yield stress,

$\mu[\mathrm{Pa} \cdot \mathrm{s}]$ is dynamic viscosity,

$\dot{\gamma}\left[\mathrm{s}^{-1}\right]$ expresses shear rate.

The shear yield stress is considered as constant commonly. However, in this case, it is a function of many variables and it is defined by the following equation:

$$
\tau_{y}=k_{y} \cdot B^{n_{y}}
$$

Where $B$ is magnetic induction,

$k_{y}$ and $n_{y}$ are material constant of magnetorheological fluid.

For this simulation following values were chosen $k_{y}=10000 \mathrm{~Pa} \cdot \mathrm{T}^{-2} ; n_{y}=2$. Magnetic induction is also dependent on the thickness of the lubricating film in radial direction. Definition of magnetic induction in squeeze film damper is described by the following relation:

$$
B=k_{B} \cdot \mu_{0} \cdot \mu_{r} \cdot \frac{I}{h(t)}
$$

where $k_{B}$ expresses construction parameter of damper (100 threads and efficiency $60 \%$ means its value is $k_{B}=0,6$ ),

$\mu_{0}$ is a permeability of vacuum $\left(4 \pi \cdot 10^{-7} \mathrm{Hm}^{-1}\right)$,

$\mu_{r}$ is relative permeability of magnetorheological fluid which for our case has value 5 ,

$I$ is electric current which flows through coil, which was set on value $0.2 \mathrm{~A}$ in this paper.

Time dependent size of the thickness of the lubricating film is defined by $h(t)$ :

$$
h=c-e \cdot \cos (\varphi-\gamma)
$$


where $h$ - thickness of the oil film,

c - the width of the gap between the inner and outer rings of the damper,

$e \quad$ - the eccentricity,

$\varphi \quad$ - the circumferential coordinate,

$\gamma \quad$ - the position angle of the line of centre (Fig. 3).

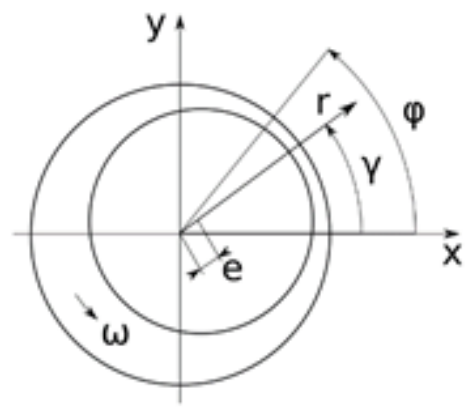

Fig. 3. Inner ring motion

\section{Solution and results}

Motion of squeeze film damper inner ring is time dependent. Therefore, the solution was simulated as transient. The size of time step has values $10^{-4} \mathrm{~s}$. The viscosity of filling fluid in damper was relatively high, velocities and the size of gap width are small. Therefore, also the Reynolds numbers come out small. And for this reason no turbulence model was used and laminar model was chosen.

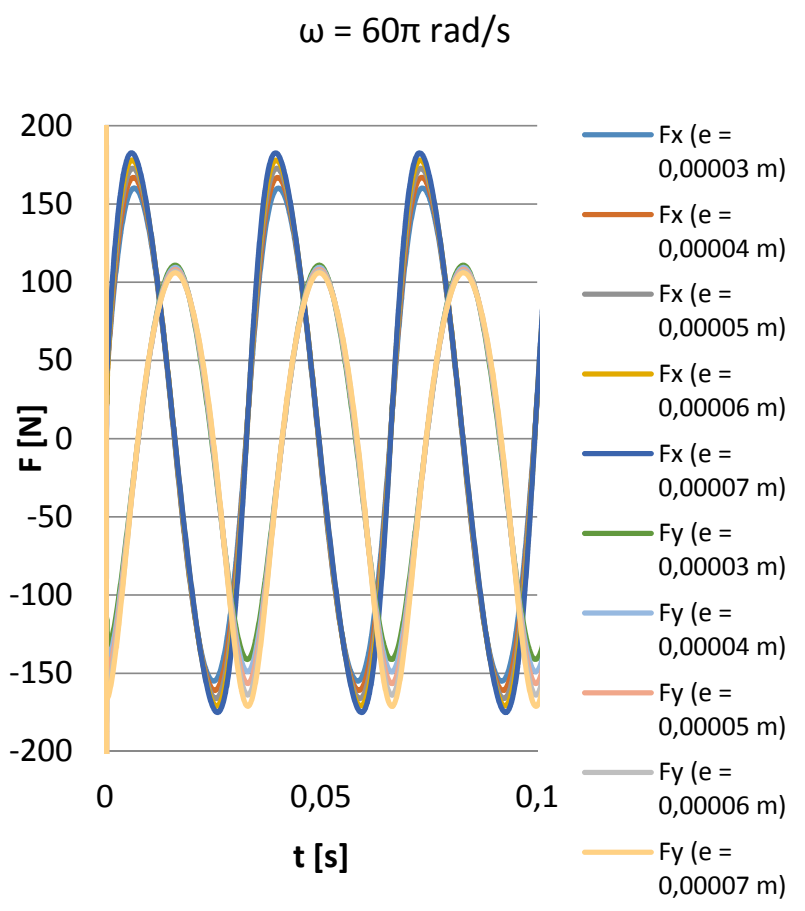

Fig. 4. Force components for $\omega=60 \pi \mathrm{rad} / \mathrm{s}$

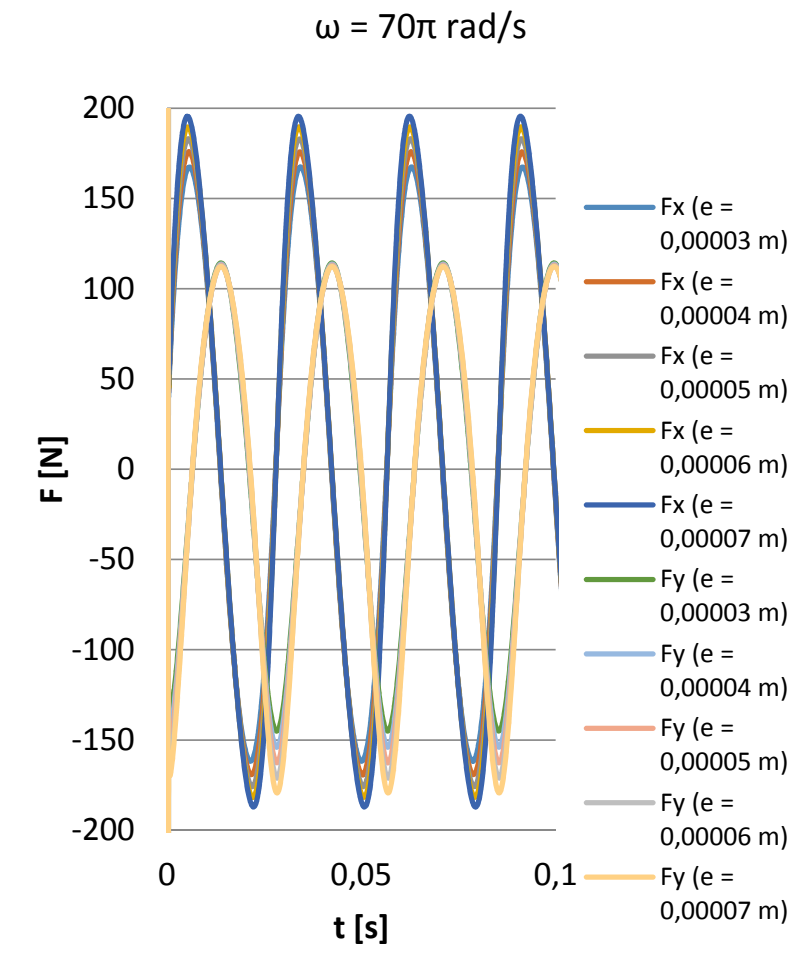

Fig. 5. Force components for $\omega=70 \pi \mathrm{rad} / \mathrm{s}$

$\omega=80 \pi \mathrm{rad} / \mathrm{s}$

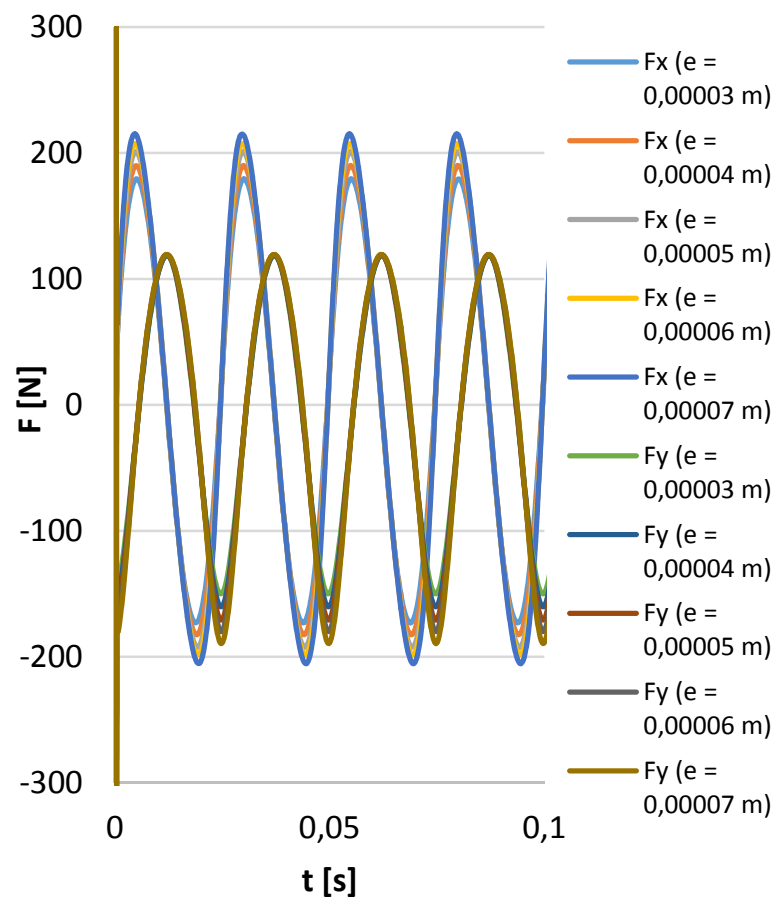

Fig. 6. Force components for $\omega=80 \pi \mathrm{rad} / \mathrm{s}$ 


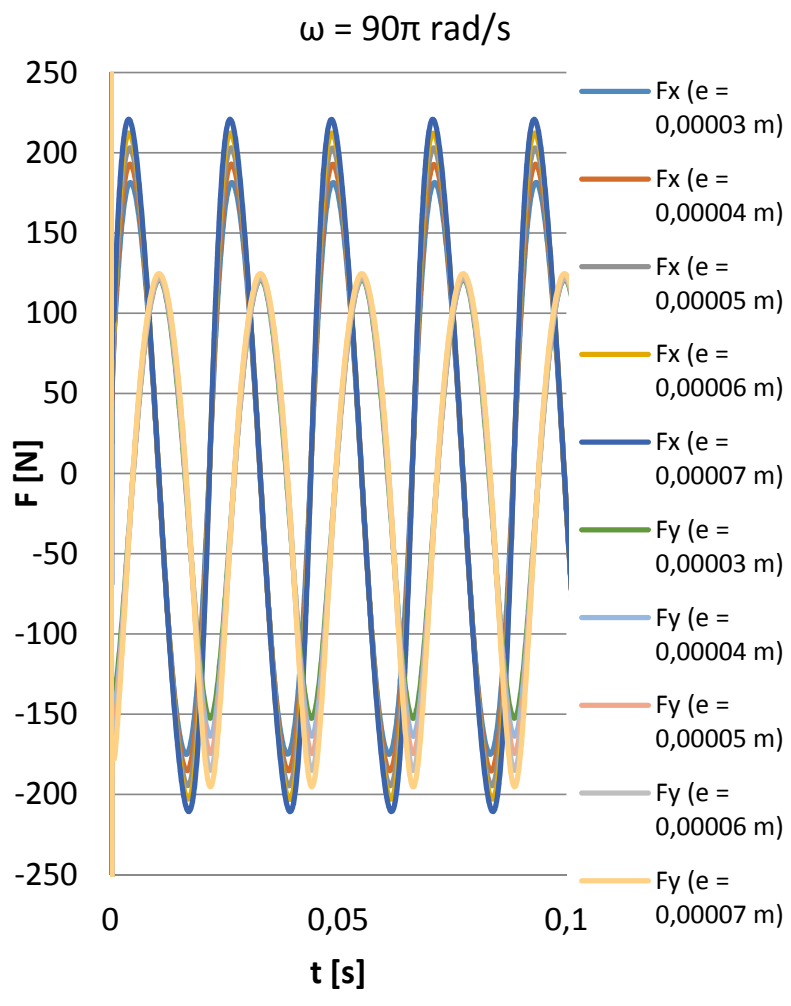

Fig. 7. Force components for $\omega=90 \pi \mathrm{rad} / \mathrm{s}$

\section{$\omega=100 \pi \mathrm{rad} / \mathrm{s}$}

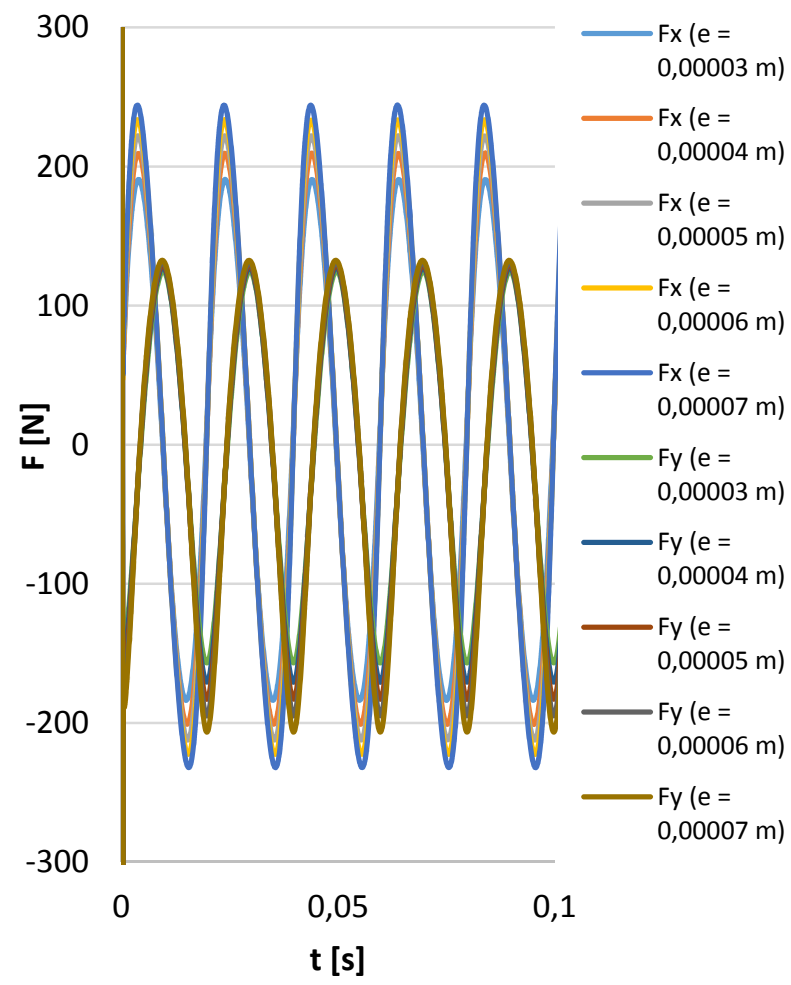

Fig. 8. Force components for $\omega=100 \pi \mathrm{rad} / \mathrm{s}$
In Fig. 4.-8. force components are displayed for different eccentricities and different angular velocities. Values are periodically changing. Values of positive and negative amplitudes of forces in $\mathrm{x}$ axis direction are almost same. That does not apply to amplitudes of forces in y axis direction as well. Positive force amplitudes in y axis direction are almost constant for one value of angular velocity, but negative values are different.

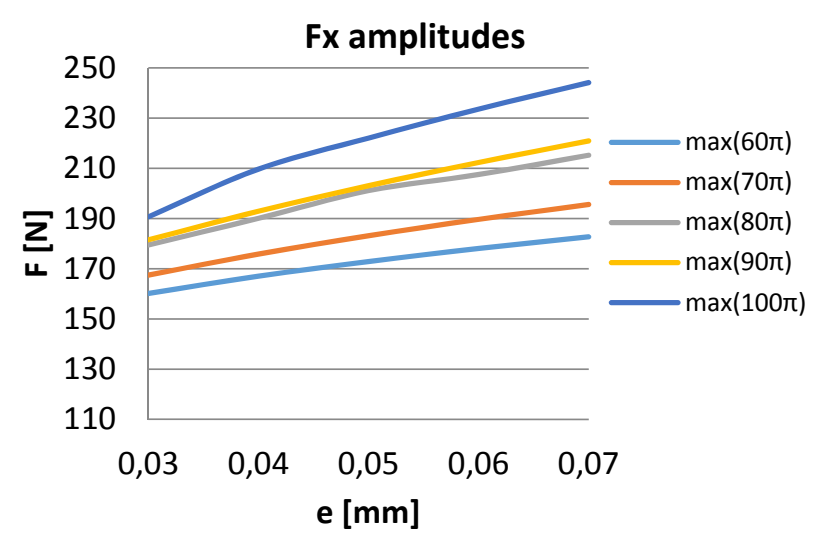

Fig. 9. Fx positive amplitudes

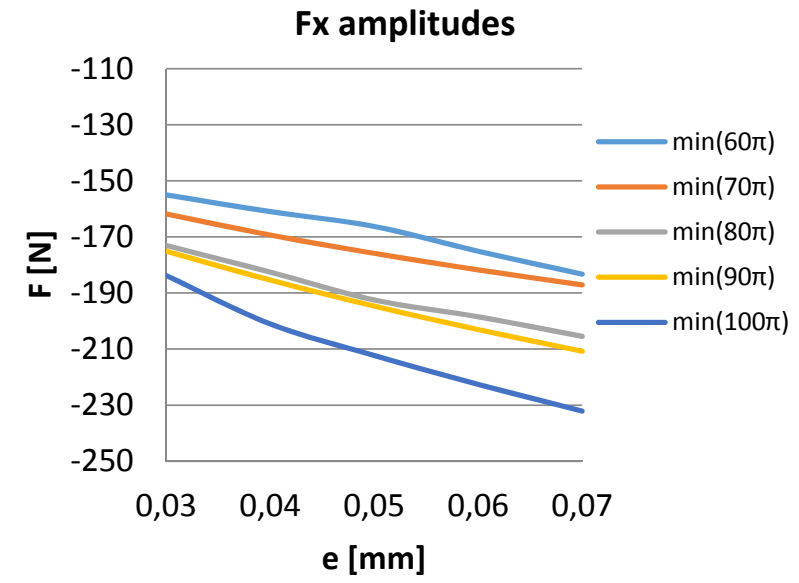

Fig. 10. Fx negative amplitudes

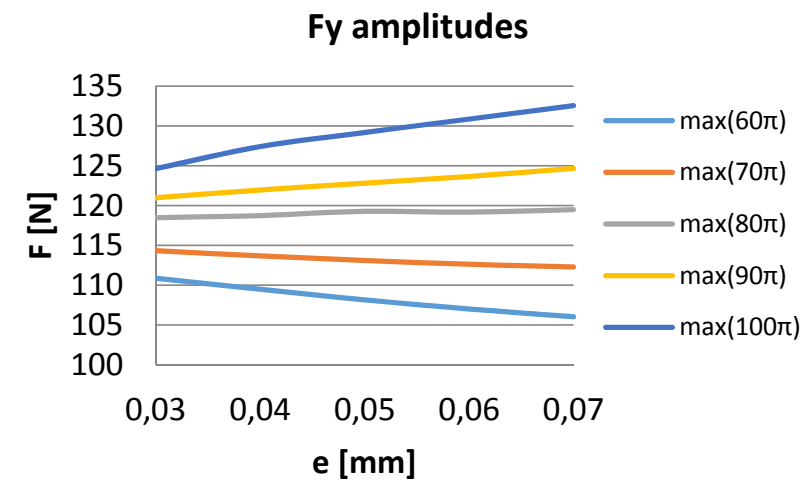

Fig. 11. Fy positive amplitudes 


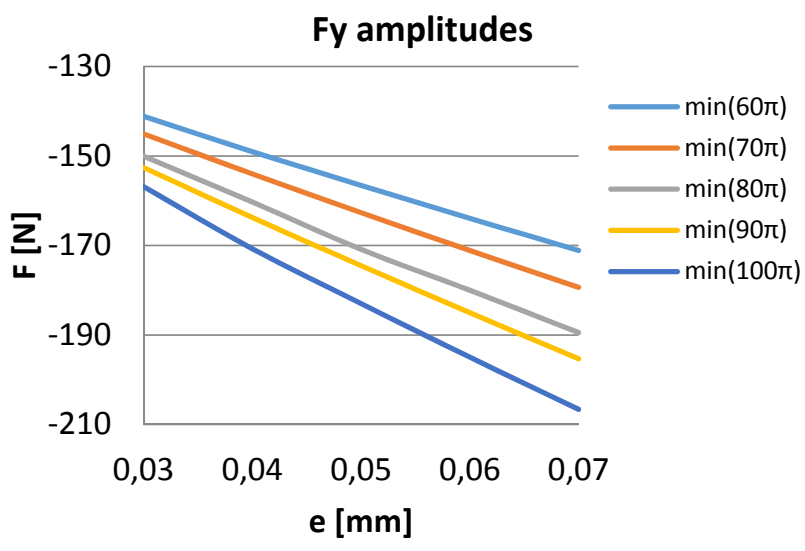

Fig. 12. Fy negative amplitudes

Values of force amplitudes as function of amplitude of deviation are displayed in Fig. 9. - 12. Values of force amplitudes in direction of $\mathrm{x}$ axis are increasing with increasing values of angular velocities and with increasing values of amplitude of deviation. The same applies to Fy negative amplitudes as well. However positive values of force amplitudes in direction of y axis do not have these trends. Fy positive amplitudes are almost constant in case, when angular velocity was equal to $80 \pi \mathrm{rad} / \mathrm{s}$. Force effects are decreasing for smaller angular velocities and increasing for higher angular velocities. Erroneous assumption was to expected that the force effects will be increasing with increasing values of angular velocities. Small differences can be caused by inaccuracy of numerical solutions. This phenomenon has to be studied in detail.

\section{Conclusions and future work}

Force effects are dependent on a size of an eccentricity of inner ring motion and its angular velocity. Force amplitudes in both direction increases with increasing motion eccentricity. However results show that description of force amplitudes on inner ring as function of angular velocity is more complicated than it was expected. This phenomenon will be studied in future work more depth. Next plan is to extend the present numerical model by new boundary condition of different wettability of surface.

\section{Acknowledgment}

Grant Agency of Czech Republic, within the project GA1506621S, and Faculty of mechanical engineering BUT, within the project FSI-S-17-4615, are gratefully acknowledged for support of this work.

\section{References}

1. Zapoměl, J.; P. Ferfecki a P. Forte. A computational investigation of the transient response of an unbalanced rigid rotor flexibly supported and damped by short magnetorheological squeeze film dampers. Smart Materials and
Structures. 2012, 21(10), 105011-. DOI: 10.1088/0964-1726/21/10/105011. ISSN 09641726.

2. Zapomněl, J.; Ferfecki, P.; Kozánek, J. Mathematical modelling of a damping element working on the principle of squeezing two layers of normal and magnetorheological oils arranged in series and its application for vibration attenuation of a rigid rotor. In Náprstek, J.; Fischer, C. (ed.). Engineering Mechanics 2012. Prague: ITAM AS CR, v. v. i., 2012, s. 1581-1588. ISBN 978-8086246-40-6. [Engineering Mechanics 2012 /18./, Svratka, 14.05.2012-17.05.2012, CZ].second literature

3. Beran, J.; Bílek, M.; Žabka, P., ed. Advances in mechanism design II: Proceedings of the XII International Conference on the Theory of Machines and Mechanisms. Springer International Publishing. ISBN 978-331-9440-866. ISSN 22110984.third literature

4. Krutil, J.; Pochylý, F.; Fialová, S. (2015) Nonlinear Mathematical Model of the Rotor Motion in a Thin Hydrodynamic gap. International Science Index Conference, Amsterdam, Netherlands, 2015 proceedings, pp 305-309

5. Pochylý, F.; Fialová, S.; Malenovský, E. (2012) Bearing with magnetic fluid and hydrophobic surface of the lining. IOP Conference Series: Earth and Environmental Science. 2012. 15 (2). p. 1 - 9. ISSN 1755-1315

6. Pochylý, F.; Fialová, S.; Kozubková, M. (2010) Assessment of cavitation creation depending on the surface wettability, IOP Conference Series: Earth and Environmental Science, WOS: 000325657000106Luigi T. De Luca, Propulsion physics (EDP Sciences, Les Ulis, 2009)

7. Šedivý, D. ; Ferfecki, P. ; Fialová, S. Force Effects on Rotor of Squeeze Film Damper Using Newtonian and Non-Newtonian Fluid. Proceedings of the 36th Meeting of Departments of Fluid Mechanics and Thermomechanics. Pilsen, Czech Republic: AIP Publishing, 2017. http://aip.scitation.org/doi/abs/10.1063/1.5004368 